\title{
Neuroanatomy and Severity of Stroke in Patients with Type A Aortic Dissection
}

\author{
Akash Fichadiya ${ }^{1}$, Bijoy Menon $^{1}$, Alex Gregory ${ }^{1}$, Ericka Teleg ${ }^{1}$, and Jehangir Appoo ${ }^{1}$ \\ ${ }^{1}$ University of Calgary Cumming School of Medicine
}

July 16, 2021

\begin{abstract}
Background: Strokes are a known complication of acute type A aortic dissection (ATAAD) repair. Understanding the neuroanatomy, mechanism, and severity of stroke will facilitate efforts to improve prediction, prevention, and treatment strategies. Methods: Retrospective review of patients who sustained stroke from a consecutive series of patients undergoing ATAAD repair. Neuroimaging was interpreted by two stroke neurologists blinded to clinical results. Severity of stroke was assessed by National Institutes of Health Stroke Scale (NIHSS). Residual disability at thirty days was assessed using the modified Rankin Scale (mRS). Results: Twenty percent (38/189) of patients undergoing repair for ATAAD had stroke [unilateral 58\%, bi-hemispheric $42 \%(\mathrm{p}=0.33)$ ]. All strokes were ischemic. No significant lateralization (right vs. left) was noted with unilateral strokes $(26 \%$ vs $32 \%, \mathrm{p}=0.67)$. Etiology of stroke was embolic $(58 \%)$, hypoperfusion $(26 \%)$, mixed $(11 \%)$ and unknown $(5 \%)$. There were no intraoperative variables that correlated with the neuroanatomy or mechanism of stroke. Pre-operative carotid dissection was seen in $40 \%(n=15)$, while postoperatively $10 \%(n=4)$ sustained intracranial large vessel occlusion (LVO). Strokes were moderate or severe (NIHSS [?] 9) in $97 \%$ of cases, with $66 \%$ incidence of moderate residual disability (mRS [?] 3) at one month postoperatively. Conclusions: Stroke associated with ATAAD is heterogeneous in etiology and location. Most strokes are severe on detection and result in significant residual disability. One in 10 strokes are due to LVO amenable to endovascular treatment. Future trials may evaluate the role for early neuroimaging and treatment of incident stroke given modern advancements in endovascular stroke therapy.
\end{abstract}

\section{Hosted file}

Manuscript Final.doc available at https://authorea.com/users/425951/articles/530626neuroanatomy-and-severity-of-stroke-in-patients-with-type-a-aortic-dissection 


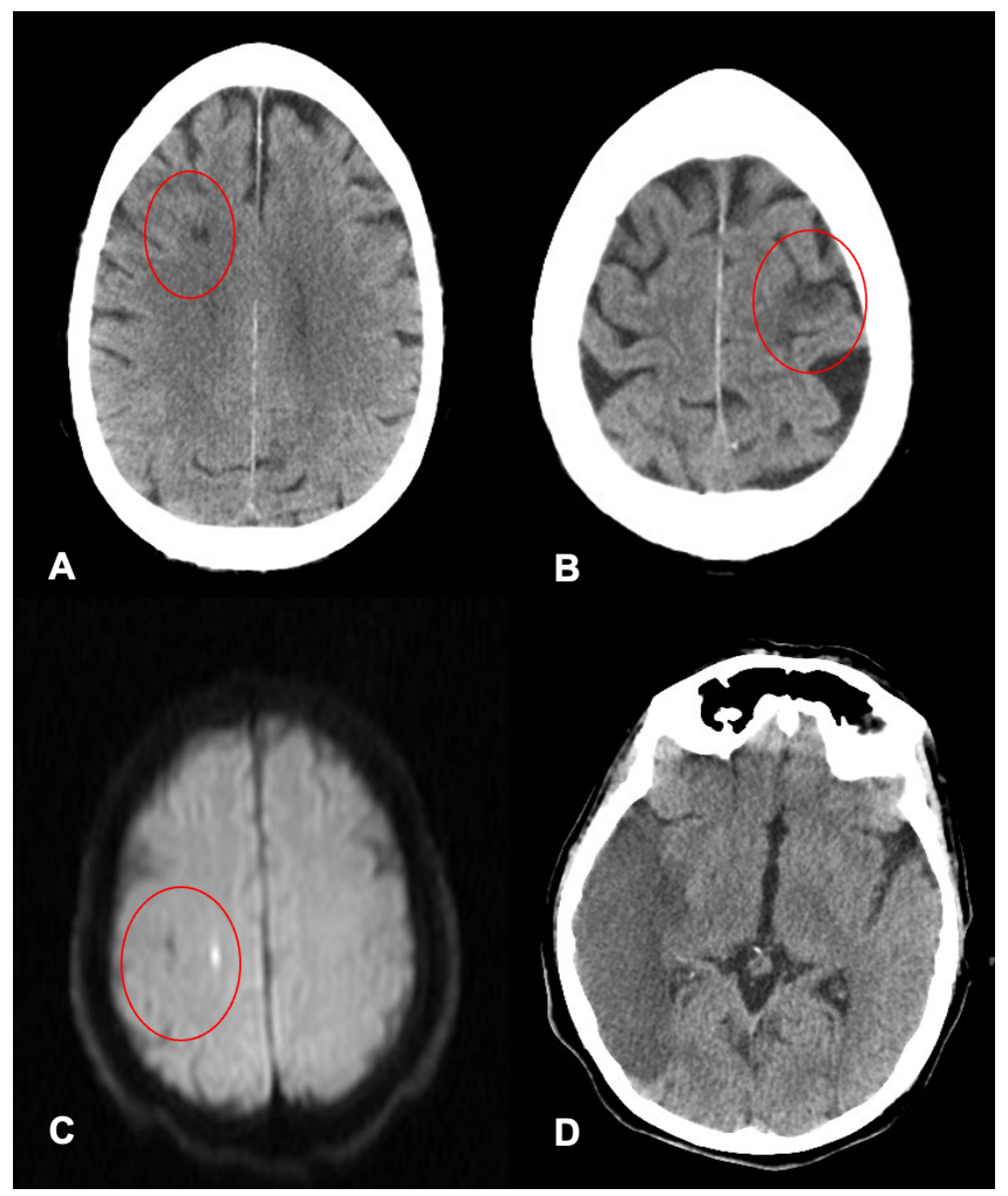


- Hypoperfusion Embolic Mixed Unknown
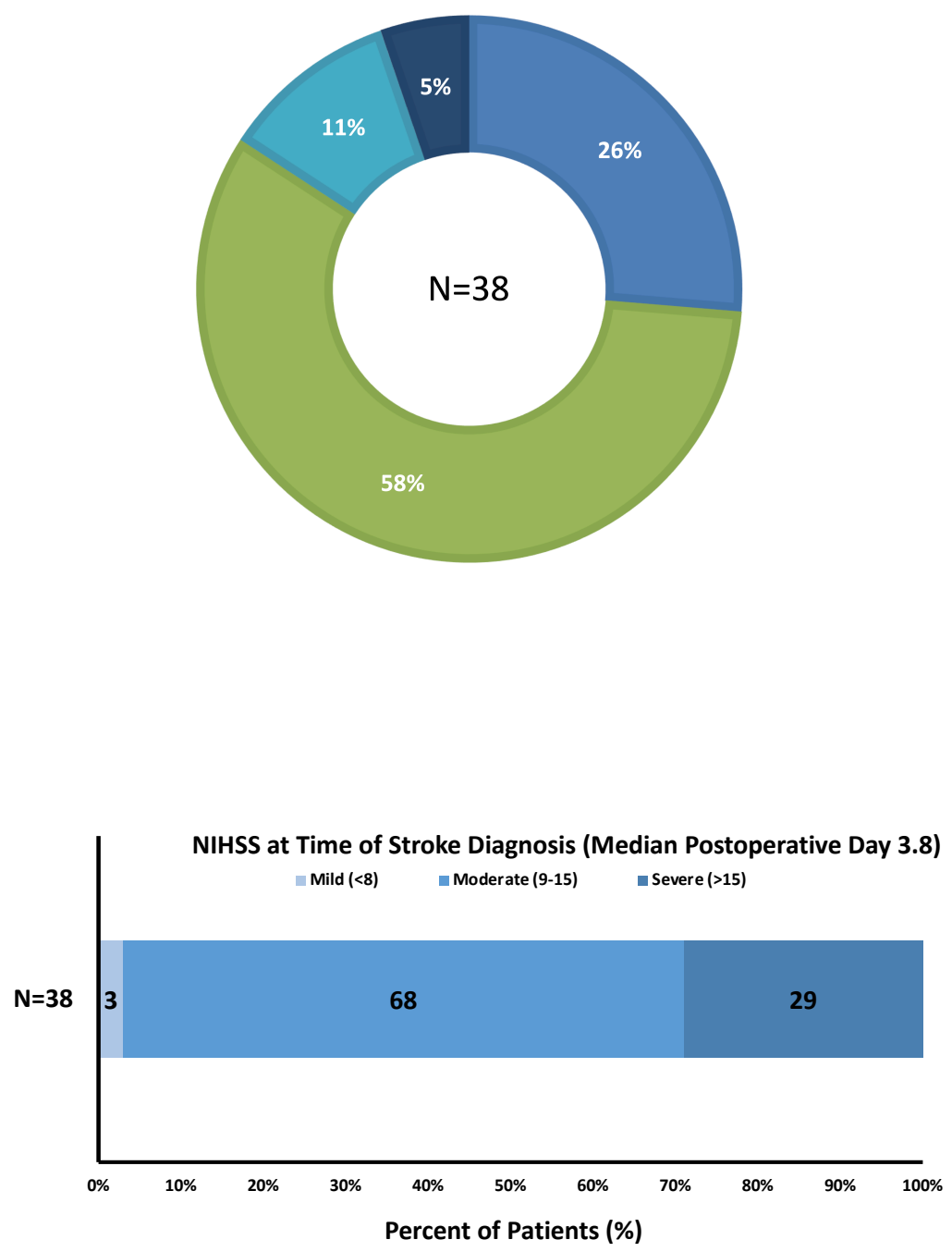

A.

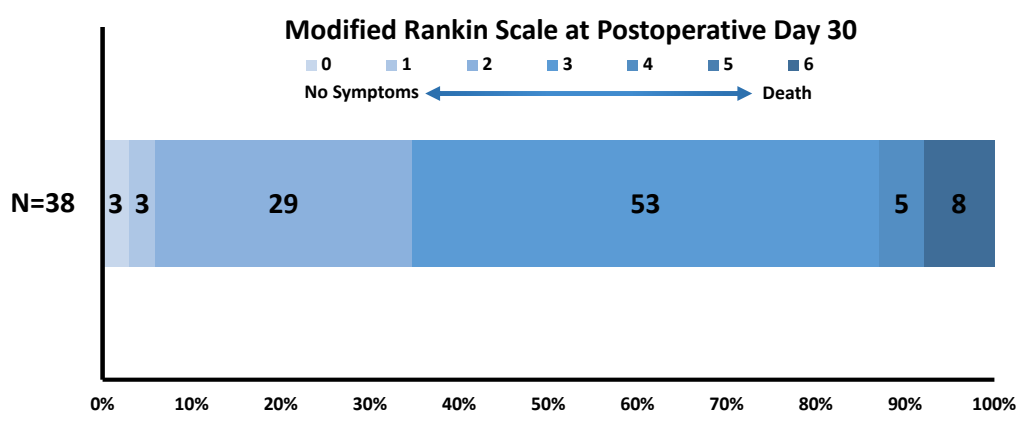

B.

Percent of Patients (\%) 